\title{
Compare the Segmentation Accuracy of Heart Substructure on Contrast Enhanced CT by Deep Convolutional Neural Network with Different Loss Functions
}

\author{
Danju Huang \\ Yunnan Cancer Hospital \\ Han Bai \\ Yunnan Cancer Hospital \\ Li Wang \\ Yunnan Cancer Hospital \\ Yu Hou \\ Yunnan Cancer Hospital \\ Lan Li \\ Yunnan Cancer Hospital \\ Yaoxiong Xia \\ Yunnan Cancer Hospital \\ Wenrui Chen \\ Yunnan Cancer Hospital \\ Zhirui Yan \\ Yunnan Cancer Hospital \\ Zhaocai Chen \\ Manteia Technologies Co.,Ltd \\ Wei Zhang \\ Manteia Technologies Co.,Ltd \\ Li Chang \\ Yunnan Cancer Hospital \\ Wenhui Li ( $\nabla$ wenhuili96@163.com ) \\ Yunnan Cancer Hospital
}

\section{Research}

Keywords: U-Net, V-Net, Loss Function, Auto segmentation, Radiotherapy

Posted Date: September 30th, 2020 
DOI: https://doi.org/10.21203/rs.3.rs-83236/v1

License: (a) (i) This work is licensed under a Creative Commons Attribution 4.0 International License. Read Full License 


\section{TITLE PAGE}

Manuscript Title : Compare the Segmentation Accuracy of Heart Substructure on Contrast Enhanced CT by Deep Convolutional Neural Network with Different Loss Functions

My manuscript is submitted as an original works: Yes, it is.

Authors: Danju Huang ${ }^{1 \#}$, Han Bai ${ }^{1 \#}$, Li Wang ${ }^{1 \#}$, Yu Hou ${ }^{1}$, Lan $\mathrm{Li}^{1}$, Yaoxiong $\mathrm{Xia}^{1}$,Wenrui Chen ${ }^{1}, \quad$ Zhirui Yan ${ }^{1}$, Zhaocai $\mathrm{Chen}^{2}$, Wei Zhang ${ }^{2}$, Li Chang ${ }^{1 *}$, Wenhui $\mathrm{Li}^{1 *}$

\#Contribute equally to this work

*Corresponding Author 
Affiliations: 1.Department of Radiation Oncology, the Third Affiliated Hospital of Kunming Medical University, Yunnan Cancer Hospital, Kunming, Yunnan, China

2. Manteia Technologies Co.,Ltd

Email addresses of authors:Danju Huang:1178446023@qq.com; Han Bai: 807235388@qq.com; Li Wang: 1632789113@qq.com; Yu Hou: 752329627@qq.com;Lan Li:347270814@qq.com;Yaoxiong Xia:1247875853@qq.com; Wenrui Chen: 1840156781@qq.com; Zhirui Yan: 704480895@qq.com; Zhaocai Chen: chenzhaocai@manteiatech.com; Wei Zhang: zhangwei@manteiatech.com

Corresponding author: Li Chang: LiChang99@aliyun.com Wenhui Li: Wenhuili96@,163.com 
(I) Conception and design: Wenhui Li, Li Chang, Danju Huang

(II) Administrative support: Wenhui Li

(III) Provision of study materials or patients: Han Bai, Li Wang, Yu Hou, Lan Li, Yaoxiong Xia

(IV) Delineation of cardiac substructure:Danju Huang, Li Wang, Yu Hou, Lan Li, Yaoxiong Xia, Wenrui Chen, Zhirui Yan,

(V) Neural network algorithm design: Danju Huang, Han Bai, Zhaocai Chen, Wei Zhang

(VI) Collection and assembly of data: Danju Huang, Zhaocai Chen, Wei Zhang

(VII) Data analysis and interpretation: Danju Huang, Wenrui Chen, Zhirui Yan

(VIII) Manuscript writing: All authors.

(IX) Final approval of manuscript: All authors.

Grant information: Ten-thousand Talents Program of Yunnan Province (Yunling scholar, Youth talent), Yunnan Provincial Training Funds for Middle-Young Academic and Technical Leader candidate, Yunnan Provincial Training Funds for High-level Health Technical Personnel (No.L-2018001). 
Key words: U-Net, V-Net, Loss Function, Auto segmentation, Radiotherapy

Tables: 2

Figures: ${ }_{2}^{2} \_$(color - Yes $)$

Compare the Segmentation Accuracy of Heart Substructure on Contrast

Enhanced CT by Deep Convolutional Neural Network with Different Loss

Functions

Danju Huang ${ }^{1 \#}$, Han Bai ${ }^{1 \#}$, Li Wang ${ }^{1 \#}$, Yu Hou ${ }^{1}$, Lan Li ${ }^{1}$, Yaoxiong Xia ${ }^{1}$, Wenrui Chen$^{1}$, Zhirui Yan', Zhaocai Chen ${ }^{2}$, Wei Zhang ${ }^{2}$, Li Chang ${ }^{1 *}$, Wenhui Li ${ }^{1 *}$

1. Department of Radiation Oncology, the Third Affiliated Hospital of Kunming Medical University, Yunnan Cancer Hospital, Kunming, Yunnan, China 
2. Manteia Technologies Co.,Ltd

\#Contribute equally to this work

*Corresponding Author

Corresponding author: Li Chang: LiChang99@aliyun.com

Wenhui Li: wenhuili96@,163.com

\begin{abstract}
Background:We aimed to compare the segmentation accuracy of heart substructure on contrast enhanced CT by deep neural network combined with different loss functions.
\end{abstract}

Methods: We collected 35 thoracic tumor patients admitted to the Department of Radiation Oncology of Yunnan Cancer Hospital. Organ-at-risks (OARs) were defined as 10 organs of cardiac substructures (pericardium, heart, left atrium, left ventricle, right atrium, right ventricle, left main stem, left anterior descending Branch, left 
circumflex branch, right coronary artery), and use the OARs manually outlined by radiation oncologists on enhanced localization $\mathrm{CT}$ as the gold standard. The automatic segmentation results of GDL U-Net, WCEGDL U-Net, ELL U-Net, and GDL V-Net are compared with the gold standard. DSC, JC, HD, VD are used as quantitative evaluation indicators.

Results:The segmentation DSC of the pericardium, heart, atrium, and ventricle of the DCNN with different loss functions all reached above 0.87. WCEGDL U-Net segmented the pericardium with DSC of 0.961 and $95 \%$ HD of $3.449 \mathrm{~mm}$; The segmentation DSC of the heart by ELL U-Net reached 0.965 , and the 95\% HD was $3.477 \mathrm{~mm}$; GDL U-Net segmentation of left atrium and right ventricle is better, DSC is 0.896 (95\% HD: 3.429mm), 0.912 (95\% HD: 4.242mm);GDL V-Net has better segmentation performance for right atrium and left ventricle, with DSC of 0.881 (95\% HD: $3.904 \mathrm{~mm})$ and 0.940 (95\% HD: $2.821 \mathrm{~mm})$.

Conclusions: The DCNN proposed in this study have achieved better segmentation effects on the pericardium, heart and four chambers in cardiac substructure segmentation.

Keywords: U-Net, V-Net, Loss Function, Auto segmentation, Radiotherapy

\section{Background}


Radiotherapy is currently used to treat up to $50 \%$ of cancer patients, which can significantly reduce cancer recurrence and mortality (1-3). In recent years, with the improvement of treatment methods and the prolonged survival of patients, cardiovascular disease has become the most common non-malignant cause of death in patients with Hodgkin's lymphoma and breast cancer receiving radiotherapy (4-6). Radiation-induced heart disease (acute pericarditis, constrictive pericarditis, restrictive cardiomyopathy, coronary artery disease, valvular disease, heart failure, conduction abnormalities, etc.) will seriously affect the life quality of patients(7). The heart is an important multi-chambered organ. The manual segmentation method depends on the doctor's knowledge and experience, which is very time-consuming and difficult to ensure accuracy, consistency and repeatability. Traditional machine learning techniques have shown good performance on heart image segmentation (8-9). However, they usually require a large amount of feature engineering knowledge or prior knowledge to obtain satisfactory accuracy, and their segmentation results for adjacent organs with inconspicuous gray gradients are not good enough, and the automatic segmentation runs longer (10-11).

In recent years, with the emergence of full convolutional neural network (FCN) (12), there have been more and more researches on automatic segmentation of target areas and OARs based on deep convolutional neural network (DCNN), and it can get a more ideal detection and segmentation effect in the target volume and OARs (13-19). At present, most of the research on the automatic segmentation of cardiac substructures are single-organ segmentation, mainly focusing on a single atrium or 
ventricle. The basic images used for segmentation are mainly cardiac magnetic resonance imaging, coronary artery imaging CT and echocardiography (20-22). This research established the new DCNN models by adding different loss functions to the existing neural network structures U-Net (23) and V-Net (24). 10 kinds of OARs, including heart contour and heart substructure, are automatically segmented on the contrast-enhanced positioning CT used in clinical radiotherapy, and we use clinical data to verify their segmentation accuracy, in order to achieve rapid and accurate segmentation of cardiac OARs.

\section{Methods}

\subsection{Patients and data}

Retrospectively found 35 patients with thoracic tumors (including 20 breast cancer, 11 lung cancer, and 4 esophageal cancer) admitted to the Department of Radiotherapy of Yunnan Cancer Hospital from May 1, 2020 to June 30, 2020.The male to female ratio of these patients was 1:2.5, and the average age was 47.45 years. Each patient has a set of chest contrast-enhanced positioning CT (16-row 32-slice Siemens spiral CT scan, range: from the entrance of the thoracic cavity to the diaphragmatic surface of the heart, covering the outer 12th rib; posture: supine position, arms raised above the head, The head is tilted to the healthy side), and the scan thickness is $1.5 \mathrm{~mm}$. The organs at risk include: pericardium, heart, left atrium, left ventricle, right atrium, right ventricle, left main stem, left anterior descending branch, left circumflex branch, and 
right coronary artery. In this study, 35 patients were randomly divided into 29 in the training set, 3 in the validation set, and 3 in the test set. The study was conducted in accordance with the Declaration of Helsinki (as revised in 2013). The study was approved by People's committee of the Third Affiliated Hospital of Kunming Medical University.

\subsection{FCN selection}

Among various FCN architectures, U-net and V-net are the more popular FCN models and have been widely used in the field of medical image processing (25-27). The U-net model is a pixel-to-pixel, end-to-end FCN, which mainly includes a contraction path that captures context information and a symmetrical expansion path that allows precise positioning. This method can obtain accurate segmentation results while using a small amount of data for end-to-end training (23). V-net is a three-dimensional image segmentation method based on a three-dimensional FCN network. The left part of the network is composed of compression paths, while the right part decompresses the signal until it reaches its original size. The main advantage is the ability to perform voxel error back propagation during the training phase, generate segmented patches with the same size as the input patch during the test, and be able to predict the segmentation of the entire 3D image (24).

\subsection{Loss functions}

2.3.1 generalized dice loss(GDL):GDL can alleviate the serious class imbalance that 
may occur between candidate tags when targeting rare observations during the segmentation process. The formula is as follows:

$G D L=1-2 \frac{\sum_{l=1}^{2} \omega_{l} \sum_{\mathrm{n}} r_{\mathrm{ln}} p_{\mathrm{ln}}}{\sum_{l=1}^{2} \omega \sum_{n} r_{\mathrm{ln}}+p_{\mathrm{ln}}}$

Among them, rln is the standard value (GT) of the category 1 at the nth pixel, and pln is the corresponding predicted probability value. The most important thing here is wl, which is the weight of each category (28).

2.3.2 Weighted Cross-Entropy loss(WCE):WCE based on class weight and distance transformation weight is a commonly used loss function for various tasks, mainly used to measure the difference information between two probability distributions, can enhance U-Net and V-Net to learn border features(29 -30), the formula is as follows :

$$
W C E=-\frac{1}{\mathrm{~N}} \sum_{\mathrm{n}=1}^{N} \mathrm{wr}_{n} \log \left(p_{n}\right)+\left(1-r_{n}\right) \log \left(1-p_{n}\right)
$$

2.3.3 Exponential Logarithmic Loss (ELL) (31): exponential logarithmic loss which balances the labels not only by their relative sizes but also by their segmentation difficulties.

$$
L_{E X P}=W_{\text {dice }} * L_{\text {dice }}+W_{C \text { ross }} * L_{\text {Cross }}
$$

2.3.4 WCEGDL:It is a hybrid loss function that combines WCE and GDL in the same network, which increases the segmentation performance of the network structure while increasing its edge feature learning ability. 


\subsection{Image segmentation}

In this experiment, we asked an experienced and trained radiation oncologist to delineate ten OARs on contrast-enhanced positioning CT, and invited three senior radiation oncologists with rich experience to proofread. The manually sketched content is regarded as the gold standard. We add GDL, WCEGDL, Exponential Logarithmic Loss to the U-Net neural network structure to generate three kinds of DCNN: GDL U-Net, WCEGDL U-Net, ELL U-Net, and add GDL to the V-Net structure to generate a DCNN : GDL V-Net, and use the above four DCNNs to automatically segment cardiac substructures, and compare the segmentation results with the gold standard.

\subsection{Evaluation Criteria}

Take the contour volume of OARs manually drawn by the doctor as the reference value, and use the four indicators of Dice Similarity Coefficient (DSC) (32), Jaccard Coefficient (JC), Hausdorff Distance (HD) (33), and Volume Difference (VD) to quantitatively evaluate the OARs accuracy obtained by the automatic drawing method (34-35).

2.5.1 Dice Similarity Coefficient (DSC): used to evaluate the coincidence between the two sets of R and G. The calculation formula is:

$$
D C(R, G)=\frac{2|R \cup G|}{|R|+|G|}
$$

$\mathrm{R}$ is the result of automatic drawing; $\mathrm{G}$ is the result of manual drawing, that is, ground 
truth. The value ranges from 0 to 1 . The closer to 1 , the better the coincidence of the two delineation methods.

2.5.2 Jaccard Coefficient(JC): used to measure the similarity between the two sets of $\mathrm{R}$ and $\mathrm{G}$, the calculation formula is:

$$
J C(R, G)=\frac{|R \cap G|}{|R \cup G|}=\frac{|R \cap G|}{|R|+|G|-|R \cap G|}
$$

2.5.3 Hausdorff Distance(HD): it is used to measure the surface distance of R and $\mathrm{G}$ in three-dimensional space. The calculation formula is:

$$
\begin{aligned}
& H D(R, G)=\max (\mathrm{h}(R, G), \mathrm{h}(G, R)) \\
& \mathrm{h}(R, G)=\max (\min \mathrm{r}-\mathrm{g}) \\
& \mathrm{r} \in R, \mathrm{~g} \in G
\end{aligned}
$$

2.5.4 Volume Difference(VD):it refers to the percentage of the difference between the segmentation result volume and the ground truth volume in the true value volume. The closer to 0 the better, the calculation formula is:

$V D(R, G)=\frac{|R|-|G|}{|G|}$

\section{Results}

\subsection{Delineate comparison example}

A total of 35 patients were enrolled in this study, and they were randomly divided into 29 cases in the training set, 3 cases in the validation set, and 3 cases in the test set. 
The ten OARs outlined by the radiation oncologist are shown in Figure 1. The result of U-Net combined with GDL for automatic segmentation is shown in Figure 2. Experienced radiation oncologists generally believe that the results of automatic segmentation can be adjusted to meet the requirements of clinical radiotherapy.

\subsection{Segmentation}

The results of the four DCNN segmentations were compared with the gold standard manually outlined by the radiation oncologist (table 1-2).It is generally believed that a DSC value greater than 0.7 indicates that the repeatability of the two contours is acceptable and the segmentation effect is acceptable (36-37).In this study, the segmentation DSC of the pericardium, heart and four chambers of the DCNN network combined with different loss functions reached above 0.87 , and the segmentation DSC of the pericardium by WCEGDL U-Net reached 0.961 , and the $95 \%$ HD was 3.449mm; The segmentation DSC of the heart by ELL U-Net reached 0.965, 95\% HD was $3.477 \mathrm{~mm}$; GDL U-Net segmented the left atrium and right ventricle better, with DSC of 0.896 (95\% HD: 3.429mm), 0.912( 95\% HD:4.242mm); GDL V-Net has better segmentation performance for right atrium and left ventricle, DSC is 0.881 (95\% HD:3.904mm), $0.940(95 \%$ HD:2.821mm). The segmentation DSC of small organs did not reach above 0.7 , of which only the segmentation for LAD was closer to 0.7 .

3.3 Processing time

OARs' delineation is time-consuming work for oncologists. The average time for an 
experienced radiation oncologist to manually delineate ten organs at risk on a set of patients' CT is over 1 hour, while the segmentation time of the automatic segmentation method used in this study is less than 2 minutes.On the basis of automatic segmentation, the time for fine-tuning to reach the clinical treatment level is within 5 minutes.

\section{Discussion}

OARs delineation is a key step before radiotherapy planning. Precise treatment delivery relies heavily on accurate OARs delineation(38). At present, there have been many researches on automatic heart segmentation, but most of them are carried out on high-quality and well-displayed images such as MRI and CTA (39-42).This research is carried out on radiotherapy positioning CT, which is the basic image of clinical radiotherapy. The image quality is relatively low and it is difficult to identify the border of the organ. We combined different loss functions into different deep neural networks, and compared their segmentation effects.Tran (43) et al. used U-Net-based CNN to segment the right ventricle on cardiac MRI, and obtained an average DSC of 0.9 (95\%HD: 5.1mm). In this study, GDL U-Net segmented the right ventricle with an average DSC of 0.912 (95\% HD: $4.242 \mathrm{~mm}$ ), which is relatively better than Tran's segmentation results.In addition, most of the current researches on the automatic segmentation of the heart are single-organ segmentation, and only a few researches on the automatic segmentation of the heart and two ventricles. We are the first to use a 
neural network combining different loss functions to automatically segment the ten substructures of the heart.The retrospective analysis of Chen et al. (10) verified the previously appeared cardiac biventricular segmentation algorithm on the same data set. Although we did not verify it on this data set, as far as the existing segmentation results are concerned, our segmentation accuracy is better than most of the neural networks. The segmentation of coronary arteries is not ideal. This is because the training of very small target areas (the number of pixels below 30) in deep learning is very difficult, and the loss function used in this study has a strong correlation with the Dice formula. At the same time, it is easy to cause fluctuations in the Dice value due to small deviations in the delineation. The heart is a multi-chambered organ, and its delineation is very time-consuming. We believe that even if the result of automatic segmentation cannot be directly used for clinical treatment, it can be used as the initial outline. Radiation oncologists can fine-tune on the basis of automatic segmentation to meet the requirements of clinical treatment.

Although,we still have some limitations in our study. The number of people enrolled in this study was not large enough, and no external data set was used for verification. The performance of automatic segmentation for small organs is poor. In the future, the algorithm can be optimized, using different training parameters, from large to small, multi-step segmentation, and other algorithms can be added to fit or modify the model. 


\section{Conclusion}

The GDL U-Net, WCEGDL U-Net, ELL U-Net, and GDL V-Net proposed in this study have achieved better segmentation effects on the pericardium, heart and four chambers in cardiac substructure segmentation.Its segmentation performance is better than most algorithms reported in other literature.

\section{Abbreviation}

CT: Computed tomography; OARs: Organ-at-risks; GDL: Generalized dice loss; WCEGDL: Weighted Cross-Entropy loss and generalized dice loss; ELL: Exponential Logarithmic Loss; DSC:Dice Similarity Coefficient; JC:Jaccard Coefficient; HD:Hausdorff Distance; VD:Volume Difference; DCNN: Deep Convolutional Neural Network; DCNNs:Deep Convolutional Neural Networks; FCN: Fully convolutional neural; MRI: Magnetic resonance imaging; CTA: Computed tomography angiography

\section{Declaretions}

\section{Ethics approval and consent to participate}


The experimental protocol was established, according to the ethical guidelines of the Helsinki Declaration (as revised in 2013) and was approved by People's committee of the Third Affiliated Hospital of Kunming Medical University. Written informed consent was obtained from individual or guardian participants. We have read and understood your journal' s policies, and we believe that neither the manuscript nor the study violates any of these.

Consent for publication: Not applicable.

\section{Availability of supporting data:}

All data generated or analysed during this study are included in this published article.

\section{Competing interests:}

The authors declare that they have no competing interests.

\section{Funding:}

This study was supported in part by grants from Ten-thousand Talents Program of Yunnan Province (Yunling scholar, Youth talent), Yunnan Provincial Training Funds for Middle-Young Academic and Technical Leader candidate, Yunnan Provincial 
Training Funds for High-level Health Technical Personnel (No.L-2018001).

\section{Authors' contributions:}

(I) Conception and design: Wenhui Li, Li Chang, Danju Huang

(II) Administrative support: Wenhui Li

(III) Provision of study materials or patients: Han Bai, Li Wang, Yu Hou, Lan Li, Yaoxiong Xia

(IV) Delineation of cardiac substructure:Danju Huang, Li Wang, Yu Hou, Lan Li, Yaoxiong Xia, Wenrui Chen, Zhirui Yan,

(V) Neural network algorithm design: Danju Huang, Han Bai, Zhaocai Chen, Wei Zhang

(VI) Collection and assembly of data: Danju Huang, Zhaocai Chen, Wei Zhang

(VII) Data analysis and interpretation: Danju Huang, Wenrui Chen, Zhirui Yan

(VIII) Manuscript writing: All authors.

(IX) Final approval of manuscript: All authors.

Acknowledgements: Not applicable. 
Author details: 1.Department of Radiation Oncology, the Third Affiliated Hospital of Kunming Medical University, Yunnan Cancer Hospital, Kunming, Yunnan, China

\section{References}

1. Boice JD Jr. An affair of the heart. J Natl Cancer Inst. 2007;99:186-7.

2. Fermé C, Eghbali H, Meerwaldt JH, Rieux C, Bosq J, Berger F, et al. Chemotherapy plus involved-field radiation in early-stage Hodgkin's disease. N Engl J Med. 2007;357:1916-27.

3. Fisher B, Anderson S, Redmond CK, Wolmark N, Wickerham DL, Cronin WM. Reanalysis and results after 12 years of follow-up in a randomized clinical trial comparing total mastectomy with lumpectomy with or without irradiation in the treatment of breast cancer. N Engl J Med. 1995;333:1456-61.

4. Aleman BM, van den Belt-Dusebout AW, Klokman WJ, Van't Veer MB, Bartelink H, van Leeuwen FE. Long-term cause-specific mortality of patients treated for Hodgkin's disease. J Clin Oncol. 2003;21:3431-9.

5. $\mathrm{Ng} \mathrm{AK}$. Review of the cardiac long-term effects of therapy for Hodgkin lymphoma. Br J Haematol. 2011;154:23-31. 
6. Patnaik JL, Byers T, DiGuiseppi C, Dabelea D, Denberg TD. Cardiovascular disease competes with breast cancer as the leading cause of death for older females diagnosed with breast cancer: a retrospective cohort study. Breast Cancer Res. 2011;13:R64.

7. Armanious MA, Mohammadi H, Khodor S, Oliver DE, Johnstone PA, Fradley MG. Cardiovascular effects of radiation therapy. Curr Probl Cancer. 2018;42:433-42.

8. Petitjean C, Zuluaga MA, Bai W, Dacher JN, Grosgeorge D, Caudron J, et al. Right ventricle segmentation from cardiac MRI: a collation study. Med Image Anal. 2015;19:187-202.

9. Peng P, Lekadir K, Gooya A, Shao L, Petersen SE, Frangi AF. A review of heart chamber segmentation for structural and functional analysis using cardiac magnetic resonance imaging. MAGMA. 2016;29:155-95.

10. Chen C, Qin C, Qiu H, Tarroni G, Duan J, Bai W, et al. Deep Learning for Cardiac Image Segmentation: A Review. Front Cardiovasc Med. 2020;7:25.

11. Dai X, Wang X, Du L, Ma N, Xu S, Cai B, et al. [Automatic segmentation of head and neck organs at risk based on three-dimensional U-NET deep convolutional neural network]. Sheng Wu Yi Xue Gong Cheng Xue Za Zhi. 2020;37:136-41.

12. Shelhamer E, Long J, Darrell T. Fully Convolutional Networks for Semantic Segmentation. IEEE Trans Pattern Anal Mach Intell. 2017;39:640-51. 
13. Men K, Chen X, Zhang Y, Zhang T, Dai J, Yi J, et al. Deep Deconvolutional Neural Network for Target Segmentation of Nasopharyngeal Cancer in Planning Computed Tomography Images. Front Oncol. 2017;7:315.

14. Ibragimov B, Xing L. Segmentation of organs-at-risks in head and neck CT images using convolutional neural networks. Med Phys. 2017;44:547-57.

15. Gibson E, Giganti F, Hu Y, Bonmati E, Bandula S, Gurusamy K, et al. Automatic Multi-Organ Segmentation on Abdominal CT With Dense V-Networks. IEEE Trans Med Imaging. 2018;37:1822-34.

16. Zhu W, Huang Y, Zeng L, Chen X, Liu Y, Qian Z, et al. AnatomyNet: Deep learning for fast and fully automated whole-volume segmentation of head and neck anatomy. Med Phys. 2019;46:576-89.

17. Jiao H, Jiang X, Pang Z, Lin X, Huang Y, Li L. Deep Convolutional Neural Networks-Based Automatic Breast Segmentation and Mass Detection in DCE-MRI. Comput Math Methods Med. 2020;2020:2413706.

18. Fatemeh Z, Nicola S, Satheesh K, Eranga U. Ensemble U-net-based method for fully automated detection and segmentation of renal masses on computed tomography images. Med Phys. 2020.

19. Kim H, Jung J, Kim J, Cho B, Kwak J, Jang JY, et al. Abdominal multi-organ auto-segmentation using 3D-patch-based deep convolutional neural network. Sci Rep. 2020;10:6204. 
20. Wu B, Fang Y, Lai X. Left ventricle automatic segmentation in cardiac MRI using a combined CNN and U-net approach. Comput Med Imaging Graph. 2020;82:101719.

21. Lindsey T, Lee JJ. Automated Cardiovascular Pathology Assessment Using Semantic Segmentation and Ensemble Learning. J Digit Imaging. 2020;33:607-12.

22. Xiong Z, Fedorov VV, Fu X, Cheng E, Macleod R, Zhao J. Fully Automatic Left Atrium Segmentation From Late Gadolinium Enhanced Magnetic Resonance Imaging Using a Dual Fully Convolutional Neural Network. IEEE Trans Med Imaging. 2019;38:515-24.

23. Ronneberger O, Fischer P, Brox T. U-Net: Convolutional Networks for Biomedical Image Segmentation. 2015.

24. Milletari F, Navab N, Ahmadi SA. V-Net: Fully Convolutional Neural Networks for Volumetric Medical Image Segmentation. 2016.

25. Zhang Z, Wu C, Coleman S, Kerr D. DENSE-INception U-net for medical image segmentation. Comput Methods Programs Biomed. 2020;192:105395.

26. Yang T, Song J, Li L, Tang Q. Improving brain tumor segmentation on MRI based on the deep U-net and residual units. J Xray Sci Technol. 2020;28:95-110.

27. Lei Y, Tian S, He X, Wang T, Wang B, Patel P, et al. Ultrasound prostate 
segmentation based on multidirectional deeply supervised V-Net. Med Phys. 2019;46:3194-206.

28. Sudre CH, Li W, Vercauteren T, Ourselin S, Cardoso MJ. Generalised Dice overlap as a deep learning loss function for highly unbalanced segmentations. 2017.

29. Zhong P, Wang D, Miao C. An Affect-Rich Neural Conversational Model with Biased Attention and Weighted Cross-Entropy Loss. 刊名. 2019. 卷(期).

30. Li H, Zhao X, Su A, Zhang H, Gu G. Color Space Transformation and Multi-class Weighted Loss for Adhesive White Blood Cell Segmentation. IEEE Access. 2020;PP:1-1.

31. Wong KCL, Moradi M, Tang H, Syeda-Mahmood T. 3D Segmentation with Exponential Logarithmic Loss for Highly Unbalanced Object Sizes. 2018. Springer, Cham.

32. Dice LR. Measures of the Amount of Ecologic Association Between Species. Ecology. 1945;26.

33. Huttenlocher DP, Klanderman GA, Rucklidge WA. Comparing Images Using the Hausdorff Distance. 1993. IEEE Computer Society.

34. Zhang F, Cui D, Wang Q, Wei L, Zhu L, Yu Y, et al. Comparative study of deep learning-versus Atlas-based auto-segmentation of organs-at-risk in tumor radiotherapy. Chinese Journal of Medical Physics. 2019, 36(12). 
35. Munir K, Elahi H, Ayub A, Frezza F, Rizzi A. Cancer Diagnosis Using Deep Learning: A Bibliographic Review. Cancers (Basel). 2019;11.

36. Zijdenbos AP, Dawant BM, Margolin RA, Palmer AC. Morphometric analysis of white matter lesions in MR images: method and validation. IEEE Trans Med Imaging. 1994;13:716-24.

37. Artaechevarria X, Munoz-Barrutia A, Ortiz-de-Solorzano C. Combination strategies in multi-atlas image segmentation: application to brain MR data. IEEE Trans Med Imaging. 2009;28:1266-77.

38. Liu Z, Liu X, Xiao B, Wang S, Miao Z, Sun Y, et al. Segmentation of organs-at-risk in cervical cancer $\mathrm{CT}$ images with a convolutional neural network. Phys Med. 2020;69:184-91.

39. Tran PV. A Fully Convolutional Neural Network for Cardiac Segmentation in Short-Axis MRI. 2016.

40. Zreik M, Leiner T, Vos BDD, Hamersvelt RWV, Isgum I. Automatic segmentation of the left ventricle in cardiac CT angiography using convolutional neural networks. 刊名. 2016. 卷(期).

41. Moccia S, Banali R, Martini C, Muscogiuri G, Pontone G, Pepi M, et al. Development and testing of a deep learning-based strategy for scar segmentation on CMR-LGE images. MAGMA. 2019;32:187-95.

42. $\mathrm{Xu} \mathrm{C}, \mathrm{Xu} \mathrm{L}$, Gao Z, Zhao S, Zhang H, Zhang Y, et al. Direct delineation of 
myocardial infarction without contrast agents using a joint motion feature learning architecture. Med Image Anal. 2018;50:82-94.

43. Tran CT, Halicek M, Dormer JD, Tandon A, Hussain T, Fei B. Fully automated segmentation of the right ventricle in patients with repaired Tetralogy of Fallot using U-Net. Proc SPIE Int Soc Opt Eng. 2020;11317.

\section{Legends}

Figure 1: The gold standard manually outlined by radiation oncologists. Green lines: pericardium; pink lines: heart; orange lines: left atrium; sky blue lines: right atrium; red lines: left ventricle; navy blue lines: right ventricle;

Figure 2: Segmentation results of U-Net with generalized dice loss function for test cases shown in transverse CT slices.Green lines: pericardium; light pink lines: heart; orange lines: left atrium; sky blue lines: right atrium; red lines: left ventricle; navy blue lines: right ventricle; 


\section{Figures}

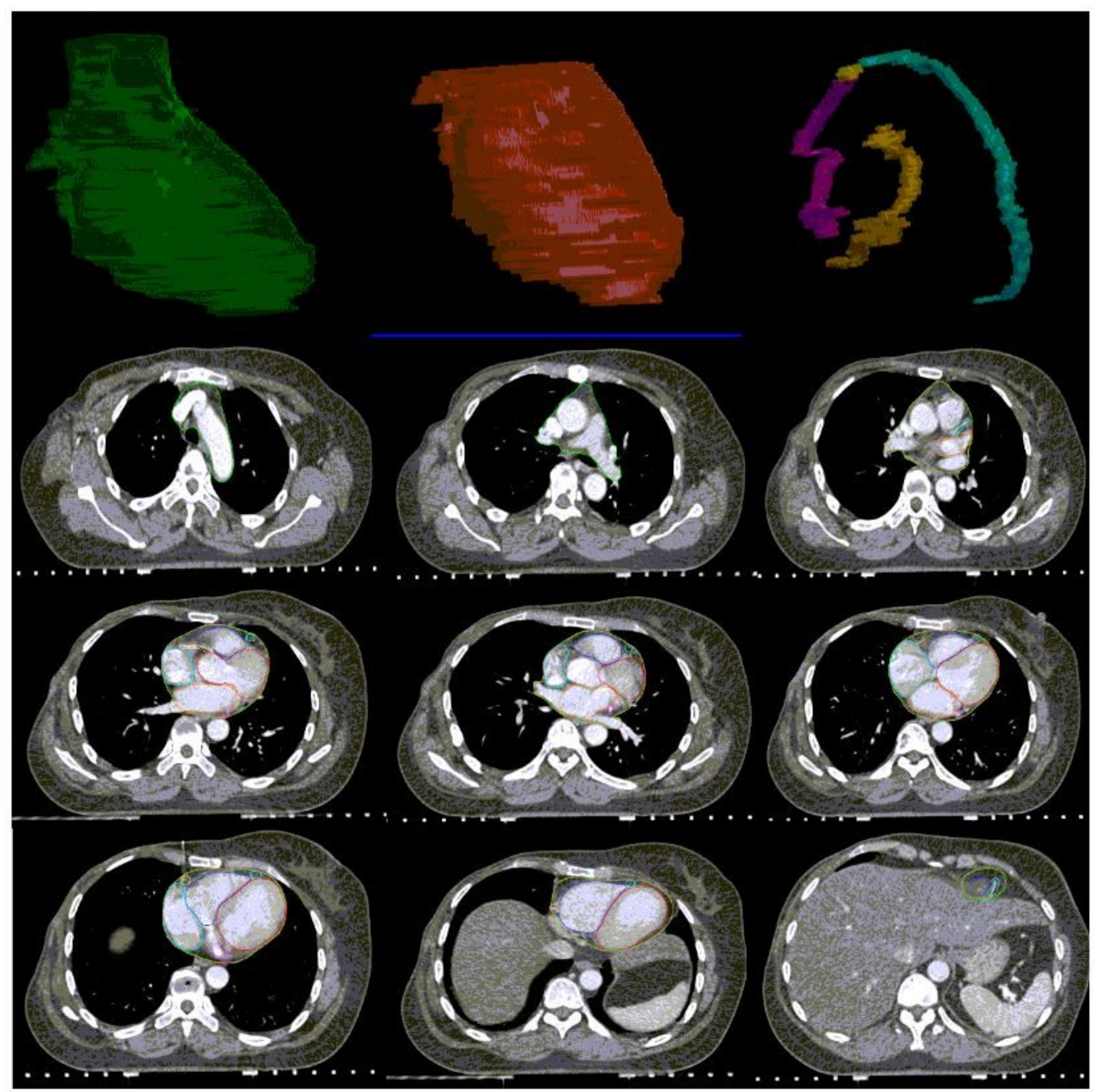

Figure 1

The gold standard manually outlined by radiation oncologists. Green lines: pericardium; pink lines: heart; orange lines: left atrium; sky blue lines: right atrium; red lines: left ventricle; navy blue lines: right ventricle; 


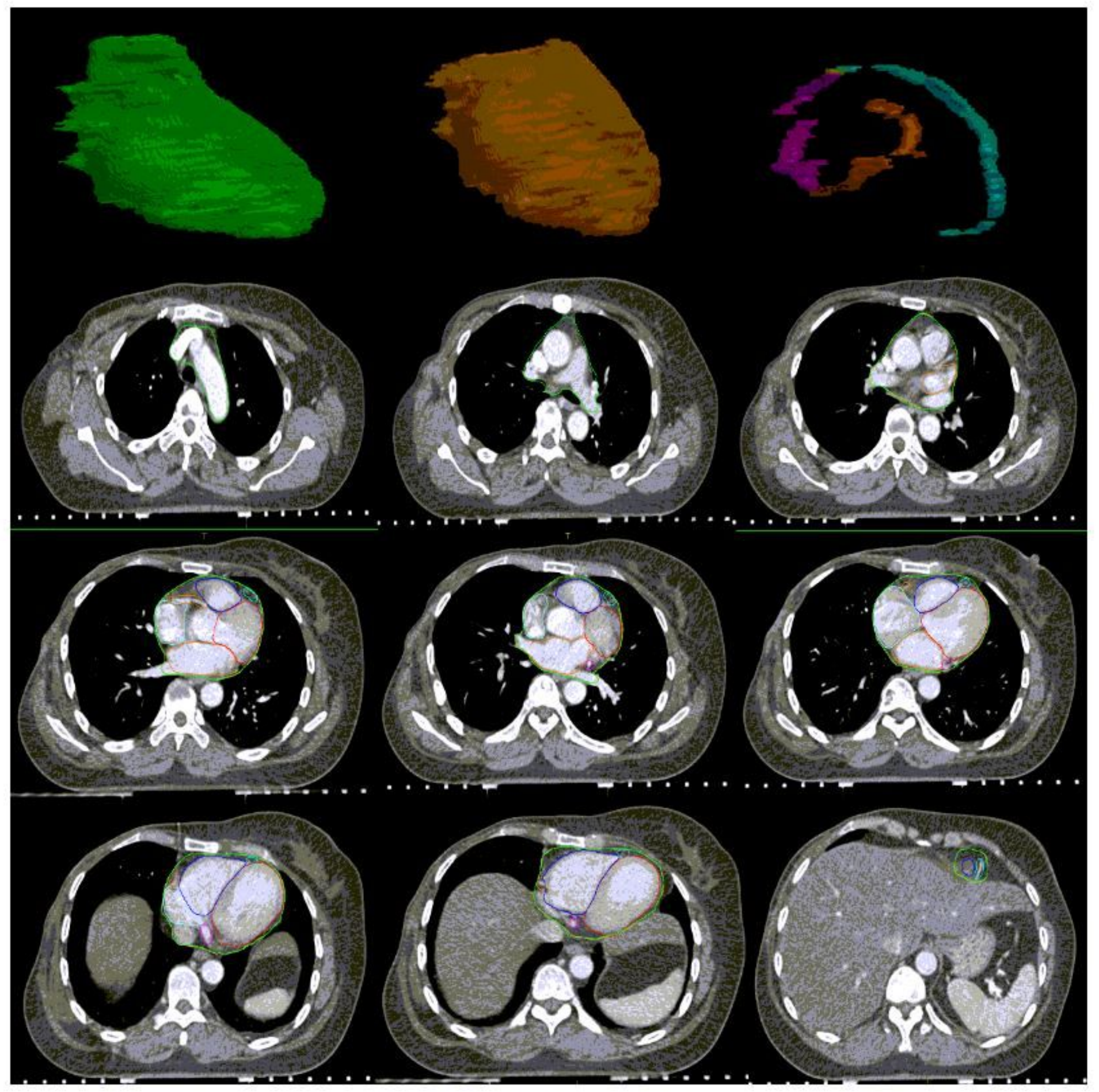

Figure 2

Segmentation results of U-Net with generalized dice loss function for test cases shown in transverse CT slices. Green lines: pericardium; light pink lines: heart; orange lines: left atrium; sky blue lines: right atrium; red lines: left ventricle; navy blue lines: right ventricle;

\section{Supplementary Files}


This is a list of supplementary files associated with this preprint. Click to download.

- table1.docx

- table2.docx 